\title{
Prototheca zopfii
}

National Cancer Institute

\section{Source}

National Cancer Institute. Prototheca zopfii. NCI Thesaurus. Code C124363.

A species of achlorophyllic alga in the family Chlorellaceae. P. zopfii is a rare opportunistic pathogen, mostly infecting dogs and cattle. 